\title{
Event-triggered nonlinear control for attitude stabilization of a quadrotor
}

\author{
J.F. Guerrero-Castellanos • \\ J.J. Téllez-Guzmán • S. Durand . \\ N. Marchand · J.U. Alvarez-Muñoz
}

Received: date / Accepted: date

\begin{abstract}
Event-triggered control is a resource-aware sampling strategy that updates the control value only when a certain condition is satisfied, which denotes event instants. Such a technique allows to reduce the control computational cost and communications. In this paper, a quaternion-based feedback is developed for event-triggered attitude stabilization of a quadrotor mini-helicopter. The feedback is derived from the universal formula for eventtriggered stabilization of general nonlinear systems affine in the control. The proposed feedback ensures the asymptotic stability to the desired attitude. Real-time experiments are carried out in order to show the convergence of the quadrotor states to the desired attitude as well as the robustness with respect to external disturbances. Results show that the proposed control can reduce by $80 \%$ the communications of the embedded system without sacrificing performance of the whole system. To the best of the authors' knowledge, this is the first time that a nonlinear event-triggered controller is experimentally applied to the attitude stabilization of an unmanned aircraft system.
\end{abstract}

Keywords Event-triggered · attitude control · quaternion · quadrotor

\section{INTRODUCTION}

A cyber-physical system (CPS) is an integration of computation with physical processes. Embedded computers and networks monitor and control the physi-

J.F. Guerrero-Castellanos, J.J. Téllez-Guzmán, J.U. Alvarez-Muñoz

Autonomous University of Puebla (BUAP), Faculty of Electronics, 7200, Puebla, Mexico

Tel.: $+52-222-2295500$

Fax: +52-222-2295631

E-mail: fguerrero@ece.buap.mx

S. Durand, N. Marchand

GIPSA-lab laboratory, Control Systems Dept., SySCo team, CNRS-Univ. of Grenoble, ENSE3 BP 46, 38402 St Martin d'Hères Cedex, France 
cal processes, usually with feedback loops where physical processes affect computations and vice versa. The intersection between physical and informationdriven functions (cyber) represents a challenge and results in innovation, see [1]. For CPS, the use of digital platforms and networks emerges as an obvious trend to save space, weight and energy. However, digital implementations can result in additional challenges, like determining how frequently the control signal needs to be updated and applied such that the stability properties are still guaranteed.

Among many CPSs, Unmanned Aerial Vehicles (UAVs) have received growing interest in industrial and academic research. They may prove useful for many civilian missions such as video supervision of road traffic, surveillance of urban districts, forest fire detection or building inspection. Furthermore, among miniature rotorcraft-based UAVs, the mini quadrotor helicopter gives rise to great interest because of its high manoeuvrability, its payload capacity and its ability to hover, as explained in [2]. Such a Vertical Take-Off and Landing (VTOL) vehicle has some advantages over conventional helicopters: owing to symmetry, it is relatively simple to design and construct. In fact, the quadrotor is an under-actuated dynamic system with four input forces and six output coordinates (attitude and position). However, this system can be broken down into two subsystems, one defining the translation movement and the other one the rotation movement. These subsystems are coupled in cascade since the translational subsystem depends on the rotational one, but the rotational subsystem is independent of the translational one. Self-governing flights require the generation of low-level control signals sent to actuators as well as decision-making related to guidance, navigation. Low-level flight control is known as attitude control and it is responsible for maintaining the desired vehicle orientation. Consequently, the attitude controller design is, in itself, a challenge.

Some linear and nonlinear control techniques have been applied for the attitude stabilization of the quadrotor mini-helicopter, like for example in [3-9]. This list is of course far from being exhaustive. Actually, all proposed attitude control laws previously listed were developed in continuous time framework and their implementation under digital platforms is carried out by means of "emulation". This procedure consists in implementing a continuous time control algorithm with a constant and sufficiently small periodic sampling period. However, this approach can be constrained by hardware and reducing the sampling period to a level that guarantees acceptable closed-loop performance may be impossible. The framework for the design of stabilizing controllers via the analysis of discrete-time systems has been widely investigated for linear systems e.g. $[10,11]$. In spite of the effort carried out to extend these results to nonlinear systems, the difficulty to obtain a nonlinear discrete-time model remains an important obstacle [12]. Then, some approaches based on an approximation of the system [13] or a redesign of the control [14] where developed but it still remains complex.

In the recent years, some works addressed resource-aware implementations of the control law using event-triggered sampling, where the control value is 
updated only when some events occur. An event is usually generated by an event-function that indicates if the control signal must be updated or not. Typical event-detection mechanisms are functions on the variation of the state (or at least the output) of the system, like in [15-20]. In [21] in particular, it is proved that such an approach reduces the number of sampling instants for the same final performance. An event-triggered paradigm hence calls for resources whenever they are indeed necessary. In the same idea, an alternative approach consists in taking events related to the variation of a Lyapunov function - and consequently to the state too - between the current state and its value at the last sampling, like in [22], or in taking events related to the time derivative of the control Lyapunov function (CLF), like in $[23,24]$. In this latter case, the updates ensure the strict decrease of a CLF, and so is ensured the asymptotically stability of the closed-loop system.

Although the advantages of event-triggered control are well-motivated and theoretical results show its potential, few results in the framework of unmanned aircraft systems have been presented in literature, e.g. [25,26]. In these works linear event-triggered controllers are proposed for attitude stabilization of a 3D helicopter model. Unfortunately, these controllers only work in a limited attraction region of the state-space. More in the spirit of task scheduling and decision, one can find works dealing with hybrid modeling and control of UAVs $[27,28]$. In these works flight modes such as Take off, Landing, Hovering, and Cruising change when there is an event. Hybrid modeling and control combines continuous dynamics with discrete automata which differs of the present work.

In this paper, we develop an event-triggered non linear control strategy for the attitude stabilization of a mini quadrotor helicopter. The update policy is driven by events issuing from the time derivative of a CLF. The feedback is quaternion-based and it is derived from the universal formula for eventtriggered stabilization of general nonlinear systems affine in the control [24]. For sake of simplicity, we only consider in this paper null stabilization with initial time instant $t_{0}=0$. The proposed feedback ensures the asymptotic stability and it is smooth everywhere except at the origin. Moreover, we propose to test such a proposal on a real-time system. The idea is to show that an event-triggered scheme could reduce the number of control functions calls even in such a case where rotor blades have to be actively controlled. To the best of the authors' knowledge this is the first time that such a method is experimentally tested.

The paper is organized as follows. First, in section 2 we present some mathematical definitions and the event-based control strategy for affine in the control nonlinear systems is detailed. The quaternion notion is also introduced and the quadrotor mini-helicopter model is given. The section 3 states the problem and presents the design of the control law for the attitude stabilization. Some experimental results are presented in section 4 and discussions finally conclude the paper. 


\section{PRELIMINARIES}

In this section some facts for event-triggered stabilization of general nonlinear systems affine in the control [24] are reviewed and the system model is introduced [8].

2.1 A universal formula for event-triggered stabilization

In this paper, the study will focus on affine in the control dynamical systems defined by:

$$
\dot{x}=f(x)+g(x) u
$$

where $x \in \mathcal{X} \subset \mathbb{R}^{n}, u \in \mathcal{U} \subset \mathbb{R}^{p}$, and $f$ a Lipschitz function vanishing at the origin. For sake of simplicity, we only consider in this paper null stabilization with initial time instant $t_{0}=0$. If the system (1) admits an asymptotic stabilizing feedback $k: \mathcal{X} \rightarrow \mathcal{U}$ then there exists a Control Lyapunov Function $V: \mathcal{X} \rightarrow \mathbb{R}$, that is a smooth function, positive definite and such that:

$$
\dot{V}=\frac{\partial V}{\partial x} f(x)+\frac{\partial V}{\partial x} g(x) k(x)
$$

It is worth noting that if $k$ is assumed to be smooth, then $V$ is known to exist and to be as smooth as $k$. In the present paper, only the smoothness of $V$ is required which is less restrictive than the one of $k$.

Event-triggered feedback usually means a set of two functions:

- an event function $e: \mathcal{X} \times \mathcal{X} \rightarrow \mathbb{R}$ that indicates if one needs $(e \leq 0)$ or not $(e>0)$ to update the control value. Event function $e$ takes the current state $x$ as input and a memory $m$ of $x$ last time $e$ became negative.

- a feedback function $k: \mathcal{X} \rightarrow \mathcal{U}$. Which is used as in the classical frame.

We recall here the definition of semi-uniform Minimum Sampling Interval (MSI) event-triggered control:

Definition 1 [24] An event-triggered feedback $(k, e)$ is said to be semi-uniformly $M S I$ if for all $\delta>0$, and all $x_{0}$ in the ball of radius $\delta$ centred at the origin $\mathcal{B}(\delta)$ the inter-execution times, that is the duration between two successive events, can be below bounded by some $\underline{\tau}>0$.

Remark 1 This minimal sampling period is useful for implementation purpose but also when the feedback $k$ is discontinuous for robustness purpose [29] as this one proposed in the present paper. 
It is known that a nonlinear system of the form (1) with a semi-uniformly MSI event-based feedback $(e, k)$, the solution of (1) starting in $x_{0} \in \mathcal{X}$ at $t=0$ is defined for all positive $t$ as the solution of the differential system:

$$
\begin{aligned}
& \dot{x}=f(x)+g(x) k(m)) \\
& \left\{\begin{array}{l}
m=x \text { if } e(x, m) \leq 0, x \neq 0 \\
\dot{m}=0 \text { elsewhere }
\end{array}\right. \\
& \text { with } x(0):=x_{0} \text { and } m(0)=x(0)
\end{aligned}
$$

Theorem 2 (Event-Triggered universal formula ) If there exists a CLF for system (1), then the event-based feedback $(e, k)$ defined below is semiuniformly MSI, smooth on $\mathcal{X} \backslash\{0\}$, and such that:

$$
\frac{\partial V}{\partial x} f(x)+\frac{\partial V}{\partial x} g(x) k(m)<0, \quad x \in \mathcal{X} \backslash\{0\}
$$

where $m$ is defined in (4) and:

$$
\begin{aligned}
k_{i}(x):= & -b_{i}(x) \delta_{i}(x) \gamma(x) \\
e(x, m):=- & a(x)-b(x) k(m) \\
& \quad-\sigma \sqrt{a(x)^{2}+\bar{\theta}(x) b(x) \Delta(x) b(x)^{T}}
\end{aligned}
$$

where

$-a(x):=\frac{\partial V}{\partial x} f(x)$ and $b(x):=\frac{\partial V}{\partial x} g(x)$,

$-x \rightarrow \Delta(x):=\operatorname{diag}\left(\delta_{1}(x), \delta_{2}(x), \ldots, \delta_{p}(x)\right)$ is a smooth function of $\mathcal{X} \backslash\{0\}$ to $\mathbb{R}^{p \times p}$, positive definite on:

$$
\mathcal{S}:=\{x \in \mathcal{X} \mid\|b(x)\| \neq 0\}
$$

$-x \rightarrow \bar{\theta}(x)$ is a smooth positive function of $\mathcal{X}$ to $\mathbb{R}$, such that $\bar{\theta}(x)\|\Delta(x)\|$ vanishes at the origin, and ensuring on $\mathcal{S} \backslash\{0\}$ the inequality $a(x)^{2}+$ $\bar{\theta}(x) b(x) \Delta(x) b(x)^{T}>0$

- $\sigma$ is a control parameter in $[0,1[$,

$-\gamma: \mathcal{X} \rightarrow \mathbb{R}$ is defined by:

$$
\gamma(x):= \begin{cases}\frac{a(x)+\sqrt{a(x)^{2}+\bar{\theta}(x) b(x) \Delta(x) b(x)^{T}}}{b(x) \Delta(x) b(x)^{T}} & \text { if } x \in \mathcal{S} \\ 0 & \text { if } x \notin \mathcal{S}\end{cases}
$$

Proof Proof was given in [24]. 
2.2 Unit quaternions and attitude kinematics

Consider two orthogonal right-handed coordinate frames: the body coordinate frame, $\mathbf{E}^{b}=\left[\mathbf{e}_{1}^{b}, \mathbf{e}_{2}^{b}, \mathbf{e}_{3}^{b}\right]$, located at the center of mass of the rigid body and the inertial coordinate frame, $\mathbf{E}^{f}=\left[\mathbf{e}_{1}{ }^{f}, \mathbf{e}_{2}{ }^{f}, \mathbf{e}_{3}{ }^{f}\right]$, located at some point in the space. The rotation of the body frame $\mathbf{E}^{b}$ with respect to the fixed frame $\mathbf{E}^{f}$ is represented by the attitude matrix $R \in S O(3)=\left\{R \in \mathbb{R}^{3 \times 3}: R^{T} R=\right.$ $I$, det $R=1\}$.

The cross product between two vectors $\xi, \chi \in \mathbb{R}^{3}$ is represented by a matrix multiplication $\left[\xi^{\times}\right] \chi=\xi \times \chi$, where $\left[\xi^{\times}\right]$is the well known skew-symmetric matrix.

The $n$-dimensional unit sphere embedded in $\mathbb{R}^{n+1}$ is denoted as $\mathbb{S}^{n}=\{x \in$ $\left.\mathbb{R}^{n+1}: x^{T} x=1\right\}$. Members of $S O(3)$ are often parametrized in terms of a rotation $\beta \in \mathbb{R}$ about a fixed axis $e_{v} \in \mathbb{S}^{2}$ by the map $\mathcal{U}: \mathbb{R} \times \mathbb{S}^{2} \rightarrow S O(3)$ defined as

$$
\mathcal{U}\left(\beta, e_{v}\right):=I_{3}+\sin (\beta)\left[e_{v}^{\times}\right]+(1-\cos (\beta))\left[e_{v}^{\times}\right]^{2}
$$

Hence, a unit quaternion, $q \in \mathbb{S}^{3}$, is defined as

$$
q:=\left(\begin{array}{c}
\cos \frac{\beta}{2} \\
e_{v} \sin \frac{\beta}{2}
\end{array}\right)=\left(\begin{array}{c}
q_{0} \\
q_{v}
\end{array}\right) \in \mathbb{S}^{3}
$$

$q_{v}=\left(\begin{array}{lll}q_{1} & q_{2} & q_{3}\end{array}\right)^{T} \in \mathbb{R}^{3}$ and $q_{0} \in \mathbb{R}$ are known as the vector and scalar parts of the quaternion respectively. $q$ represents an element of $S O(3)$ through the map $\mathcal{R}: \mathbb{S}^{3} \rightarrow S O(3)$ defined as

$$
\mathcal{R}:=I_{3}+2 q_{0}\left[q_{v}^{\times}\right]+2\left[q_{v}^{\times}\right]^{2}
$$

Note that $R=\mathcal{R}(q)=\mathcal{R}(-q)$ for each $q \in \mathbb{S}^{3}$, i.e. quaternions $q$ and $-q$ represent the same physical attitude.

Denoting by $\omega=\left(\begin{array}{lll}\omega_{1} & \omega_{2} & \omega_{3}\end{array}\right)^{T}$ the angular velocity vector of the body coordinate frame, $\mathbf{E}^{b}$ relative to the inertial coordinate frame, $\mathbf{E}^{f}$, expressed in $\mathbf{E}^{b}$, the kinematics equation is given by

$$
\left(\begin{array}{c}
\dot{q}_{0} \\
\dot{q}_{v}
\end{array}\right)=\frac{1}{2}\left(\begin{array}{c}
-q_{v}^{T} \\
I_{3} q_{0}+\left[q_{v}^{\times}\right]
\end{array}\right) \omega=\frac{1}{2} \Xi(q) \omega
$$

The attitude error is used to quantify the mismatch between two attitudes. If $q$ defines the current attitude quaternion and $q_{d}$ is the desired quaternion, i.e. the desired orientation, then the error quaternion that represents the attitude error between the current orientation and the desired one is given by

$$
q_{e}=q_{d}^{-1} \otimes q=\left(q_{e_{0}} q_{e_{v}}^{T}\right)^{T}
$$

where $q^{-1}$ is the complementary rotation of the quaternion $q$ which is given by $q^{-1}=\left(\begin{array}{ll}q_{0} & -q_{v}^{T}\end{array}\right)^{T}$ and $\otimes$ denotes the quaternion multiplication [30]. 


\subsection{System model}

The quadrotor is a small aerial vehicle that belongs to the VTOL (Vertical Taking Off and Landing) class of aircrafts. It is lifted and propelled, forward and laterally, by controlling the rotational speed of four blades mounted at the four ends of a simple cross and driven by four DC Brushless motors (BLDC). On such a platform (see Fig. 1), given that the front and rear motors rotate counter-clockwise while the other two rotate clockwise, gyroscopic effects and aerodynamic torques tend to cancel each other out in trimmed flight. The rotation of the four rotors generates a vertical force, called the thrust $T$, equal to the sum of the thrusts of each rotor $\left(T=f_{1}+f_{2}+f_{3}+f_{4}\right)$. The pitch movement $\theta$ is obtained by increasing/decreasing the speed of the rear motor while decreasing/increasing the speed of the front motor. The roll movement $\phi$ is obtained similarly using the lateral motors. The yaw movement $\psi$ is obtained by increasing/decreasing the speed of the front and rear motors while decreasing/increasing the speed of the lateral motors. In order to avoid any linear movement of the quadrotor, these maneuvers should be achieved while maintaining a value of the total thrust $T$ that balances the aircraft weight. In order to model the system's dynamics, two frames are defined: a fixed frame in the space $\mathbf{E}^{f}=\left[\mathbf{e}_{1}^{f}, \mathbf{e}_{2}{ }^{f}, \mathbf{e}_{3}{ }^{f}\right]$ and a body-fixed frame $\mathbf{E}^{b}=\left[\mathbf{e}_{1}^{b}, \mathbf{e}_{2}{ }^{b}, \mathbf{e}_{3}{ }^{b}\right]$, attached to the quadrotor at its center of gravity, as shown in Fig. 1.

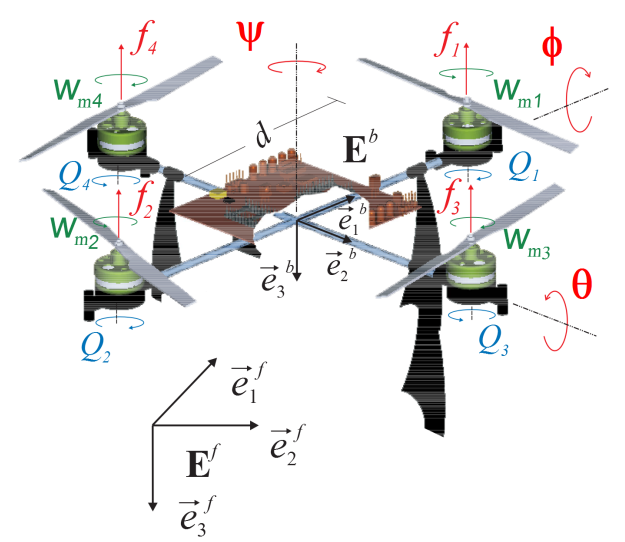

Fig. 1 Quadrotor: fixed frame $\mathbf{E}^{f}=\left[\mathbf{e}_{1}^{f}, \mathbf{e}_{2}^{f}, \mathbf{e}_{3}^{f}\right]$ and body-fixed frame $\mathbf{E}^{b}=\left[\mathbf{e}_{1}^{b}, \mathbf{e}_{2}^{b}, \mathbf{e}_{3}^{b}\right]$

According to $[31, ?]$ and 2.2, the six degrees of freedom model (position and attitude) of the system can be separated into translational and rotational motions, represented respectively by $\Sigma_{T}$ and $\Sigma_{R}$ in equation (15) and (16).

$$
\Sigma_{T}:\left\{\begin{array}{l}
\dot{p}=v \\
\dot{v}=g e_{3}-\frac{1}{m_{h}} R^{T}(q) T e_{3}
\end{array}\right.
$$




$$
\Sigma_{R}:\left\{\begin{aligned}
\dot{q} & =\frac{1}{2} \Xi(q) \omega \\
J \dot{\omega} & =-\left[\omega^{\times}\right] J \omega+\Gamma
\end{aligned}\right.
$$

where $m_{h}$ denotes the mass of the quadrotor and $J$ its inertial matrix expressed in $\mathbf{E}^{b} \cdot g$ is the gravity acceleration and $e_{3}=\left(\begin{array}{lll}0 & 0 & 1\end{array}\right)^{T} \cdot p=\left(\begin{array}{lll}x & y & z\end{array}\right)^{T}$ represents the position of the quadrotor's center of gravity, which coincides with the origin of frame $\mathbf{E}^{b}$, with respect to frame $\mathbf{E}^{f}, v=\left(v_{x} v_{y} v_{z}\right)^{T}$ its linear velocity in $\mathbf{E}^{f}$, and $\omega$ denotes the angular velocity of the quadrotor expressed in $\mathbf{E}^{b} . \Gamma \in \mathbb{R}^{3}$ depend on the couples generated by the actuators, aerodynamic couples and external couples (environmental forces). In this paper, it is assumed that these torques are only generated by the actuators. $-T e_{3}$ is the total thrust expressed in $\mathbf{E}^{b}$.

The reactive torque $Q_{j}$ due to the $j^{\text {th }}$ rotor drag, $j \in\{1,2,3,4\}$, and the total thrust $T$ generated by the four rotors can be approximated by an algebraic relationship on function of a PWM control signal applied to the BLDC-drivers:

$$
Q_{j}=k_{m} u_{m j} \quad T=b_{m} \sum_{j=1}^{4} u_{m j}=\sum_{j=1}^{4} f_{j}
$$

where the input signals $u_{m i}$ are expressed in seconds, i.e. the time during which the PWM control signal is in high state. $k_{m}>0$ and $b_{m}>0$ are two parameters that depend on the air density, the dynamic pressure, the lift coefficient, the radius and the angle of attack of the blades and they are obtained experimentally.

The components of the control torque vector $\Gamma$ generated by the rotors are given by:

$$
\begin{aligned}
& \Gamma_{1}=d b_{m}\left(u_{m 3}-u_{m 4}\right) \\
& \Gamma_{2}=d b_{m}\left(u_{m 1}-u_{m 2}\right) \\
& \Gamma_{3}=k_{m}\left(-u_{m 1}+u_{m 2}-u_{m 3}+u_{m 4}\right)
\end{aligned}
$$

with $d$ being the distance from one rotor to the center of mass of the quadrotor. Combining equations (17) and (18), the forces and torques applied to the quadrotor are written as:

$$
\begin{aligned}
\left(\begin{array}{c}
\Gamma \\
T
\end{array}\right) & =\left(\begin{array}{cccc}
0 & 0 & d b_{m} & -d b_{m} \\
d b_{m} & -d b_{m} & 0 & 0 \\
-k_{m} & -k_{m} & k_{m} & k_{m} \\
b_{m} & b_{m} & b_{m} & b_{m}
\end{array}\right)\left(\begin{array}{l}
u_{m 1} \\
u_{m 2} \\
u_{m 3} \\
u_{m 4}
\end{array}\right) \\
& =N U_{m}
\end{aligned}
$$

where $U_{m}=\left(\begin{array}{llll}u_{m 1} & u_{m 2} & u_{m 3} & u_{m 4}\end{array}\right)^{T}$. Since $N$ is an invertible matrix, the vector of signals control $U_{m}$ is easily obtained. 


\section{EVENT-BASED CONTROL STRATEGY}

\subsection{Problem statement}

The objective is to design a control law that drives the quadrotor attitude to a specified constant orientation and maintains this orientation starting from any initial condition. It follows that the angular velocity vector must be approach zero and remains null. In this paper, null stabilization is considered. Hence, the inertial coordinate frame is selected to be the desired orientation and the control objective is described by the following asymptotic condition:

$$
q \rightarrow\left( \pm 1 \quad 0^{T}\right)^{T}, \quad \omega \rightarrow 0 \text { as } t \rightarrow \infty
$$

Equation (19) represents two equilibrium points $\left(q_{0}=1, q_{v}=\left(\begin{array}{lll}0 & 0 & 0\end{array}\right)^{T}\right)$ and $\left(q_{0}=-1, q_{v}=\left(\begin{array}{lll}0 & 0 & 0\end{array}\right)^{T}\right)$. These equilibrium points represent the same equilibrium point in the physical space and they yield the same attitude matrix in equation (12). However, they represent two-point set in $\mathbb{S}^{3}$. This topological obstruction not allows to state any global property for the closed-loop system, using a continuous quaternion-based feedback [32,33].

In this study, the case $q_{d}=\left(\begin{array}{ll}1 & 0^{T}\end{array}\right)^{T}$ is considered.

On the other hand, the quadrotor is equipped of an Attitude Heading Reference Systems (AHRS) and an embedded computer system (see Fig. 2). The AHRS continuously monitors the state $x$ (attitude and angular velocity). Based on current state information and the last computed control signal, which is piecewise constant, the event-function decides when to broadcast the current state measurement over the network which is denoted by $x_{i}$. Whenever the control block receives a new state value, it updates the control law and the control signal for the actuators (PWM signals). Then, it broadcasts the control signal over the network in order to evaluate the event-triggered function and to detect a new event.

Thus, the problem consists in showing that the attitude of the quadrotor helicopter can be asymptotically stabilized by means of an event-triggered feedback as defined in section 2.1, i.e. with the control law (7) together with the event function (8). Another motivation is that other traffic exists between two successive events and after the update and broadcasting of the control signal over the network. Reducing the traffic used for control (thanks to an eventbased approach) hence allows i) to reduce traffic congestion in the network and ii) to broadcast other sensors data, for instance GPS or infrared sensors.

\subsection{Control design}

In order to stabilize the attitude of the quadrotor mini-helicopter, the subsystem $\Sigma_{R}$ in (16) is used. Defining the variables $x_{1}=q_{0} \in \mathbb{R}, x_{2}=q_{v} \in \mathbb{R}^{3}$, $x_{3}=\omega \in \mathbb{R}^{3}, \Sigma_{R}$ can be rewritten as

$$
\dot{x}=f(x)+g(x) u
$$




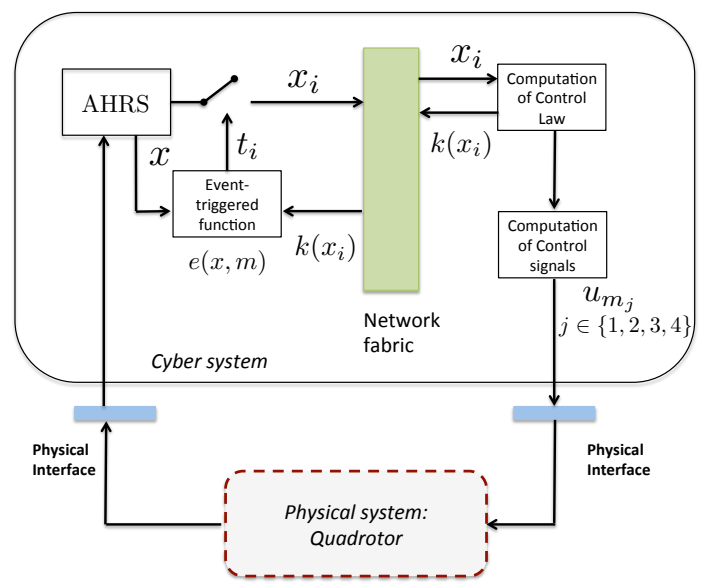

Fig. 2 Quadrotor control system

which is a nonlinear system affine in the control with state $x=\left(\begin{array}{lll}x_{1} & x_{2}^{T} & x_{3}^{T}\end{array}\right)^{T}$ control $u=\Gamma \in \mathbb{R}^{3}$ and vectors fields

$$
\begin{aligned}
& f(x)=\left(\begin{array}{c}
-\frac{1}{2} x_{2}^{T} x_{3} \\
\frac{1}{2}\left(x_{1} I_{3}+\left[x_{2}^{\times}\right]\right) x_{3} \\
-J^{-1}\left[x_{3}^{\times}\right] J x_{3}
\end{array}\right) \\
& g(x)=\left(g_{1}(x) g_{2}^{T}(x) g_{3}^{T}(x)\right)^{T}
\end{aligned}
$$

According to (19) the control objective becomes

$$
x_{0} \rightarrow 1, \quad x_{2}, x_{3} \rightarrow 0 \text { as } t \rightarrow \infty
$$

Lemma 1 The function $V: \mathbb{S}^{3} \times \mathbb{R}^{3} \longrightarrow \mathbb{R}$ defined by

$$
V=x_{2}^{T} x_{2}+\left(x_{1}-1\right)^{2}+\frac{1}{2} \tilde{x}^{T} K_{3}^{-1} J \tilde{x}
$$

with $\tilde{x}=x_{3}+K_{1} x_{2}$ is a CLF for the system (20) relative to the equilibrium state $x^{e}=\left(\begin{array}{lll}1 & 0^{T} & 0^{T}\end{array}\right)^{T}$ with the control

$$
u=\left[x_{3}^{\times}\right] J x_{3}-J K_{1} \dot{x}_{2}-K_{2} \tilde{x}-K_{3} x_{2}
$$

where $K_{1}, K_{2}, K_{3} \in \mathbb{R}^{3 \times 3}$ are diagonal positive definite matrices and $\dot{x}_{2}=$ $\frac{1}{2}\left(x_{1} I_{3}+\left[x_{2}^{\times}\right]\right) x_{3}$.

Proof Clearly $V$ is smooth, positive definite and proper. Now, consider the derivative of (23) along the trajectories of the closed-loop system with any initial condition in $\mathbb{S}^{3} \times \mathbb{R}^{3} \backslash\left(\begin{array}{lll}-1 & 0^{T} & 0^{T}\end{array}\right)^{T}$ 


$$
\begin{aligned}
\dot{V}(x) & =\frac{\partial V^{T}}{\partial x} f(x)+\frac{\partial V^{T}}{\partial x} g(x) u \\
& =x_{2}^{T} x_{3}+\tilde{x}^{T} K_{3}^{-1} J K_{1} \dot{x}_{2}+\tilde{x}^{T} K_{3}^{-1} J\left(J^{-1}\left(\left[x_{3}^{\times}\right] J x_{3}\right)\right)+\tilde{x}^{T} K_{3}^{-1} J J^{-1} u \\
& =x_{2}^{T} x_{3}+\tilde{x}^{T} K_{3}^{-1}\left(J K_{1} \dot{x}_{2}+\left[x_{3}^{\times}\right] J x_{3}+u\right) \\
& =x_{2}^{T} x_{3}-\tilde{x}^{T} K_{3}^{-1} K_{2} \tilde{x}-\tilde{x}^{T} x_{2} \\
& =x_{2}^{T} x_{3}-\tilde{x}^{T} K_{3}^{-1} K_{2} \tilde{x}-\left(x_{3}^{T}+K_{1} x_{2}^{T}\right) x_{2} \\
& =-\tilde{x}^{T} K_{3}^{-1} K_{2} \tilde{x}-K_{1} x_{2}^{T} x_{2}<0 \text { for } x \neq x^{e}
\end{aligned}
$$

Then this mean that $x_{2}, \tilde{x} \rightarrow 0$. That implies $x_{3} \rightarrow 0$ and due to the quaternion normality condition $x_{0} \rightarrow 1$. Consequently $V$ is a Control-Lyapunov Function.

Corollary 1 Consider the quadrotor mini-helicopter rotational dynamics and the CLF given by (20) and (23), respectively. Then the event-triggered feedback $(k, e)$ defined by (7) and (8) with $\bar{\theta}=x_{2}^{T} x_{2}+\left(x_{1}-1\right)^{2}$ and $\Delta(x)=I_{3}$ asymptotically stabilizes the quadrotor at $\left(\begin{array}{lll}1 & 0^{T} & 0^{T}\end{array}\right)^{T}$ with a domain of attraction equal to $\mathbb{S}^{3} \times \mathbb{R}^{3} \backslash\left(\begin{array}{lll}-1 & 0^{T} & 0^{T}\end{array}\right)^{T}$. Furthermore, the feedback $(k, e)$ is semi-uniformly MSI and smooth on $\mathbb{S}^{3} \times \mathbb{R}^{3} \backslash\left(\begin{array}{lll}1 & 0^{T} & 0^{T}\end{array}\right)^{T}$.

Proof The proof follows the one of Theorem 2.

Remark 2 Note that the stability analysis has been carried out considering the asymptotic condition $q_{d}=\left(\begin{array}{ll}1 & 0^{T}\end{array}\right)^{T}$. In the case where the asymptotic condition $q \rightarrow q_{d}$ with $q_{d} \neq\left(\begin{array}{ll}1 & 0^{T}\end{array}\right)^{T}$ is considered, the feedback becomes in function of $x_{1}=q_{e_{0}} \in \mathbb{R}, x_{2}=q_{e_{v}} \in \mathbb{R}^{3}, x_{3}=\omega \in \mathbb{R}^{3}$, where the $q_{e}$ is given by (14) which represents the attitude error between the current orientation and the desired one.

\section{EXPERIMENTAL SETUP}

This section is devoted to proving the effectiveness of the proposed eventtriggered control. Experiments on the quadrotor prototype (Fig. 3) are carried out in real-time.

This prototype is based on the mechanical structure of the 330X-S QUDFlyer developed by TSH-GAUI Hobby Corporation using four BLDC motors. The control law is executed on a Spartan-6 FPGA LX9 MicroBoard. The Spartan-6 has the ability to implement a "MicroBlaze" soft processor running at $100 \mathrm{MHz}$. Furthermore, the Spartan-6 has the advantage to develop custom modules such as PWM generators and USARTs ports. An AHRS is used to obtain the attitude quaternion and angular velocity at $73 \mathrm{~Hz}$. A Bluetooth Modem linked to a PC is used to exchange the processed data. The desired attitude $q_{d}$ is provided by means of a 5 -channel Radio-Control Spektrum DX5e 
with $2.4 \mathrm{GHz}$ radio technology. Four power modules are used to drive the motors by means of a PWM signal. The frequency of the PWM signal is fixed to $500 \mathrm{~Hz}$. The power of the whole system is supplied by a 11.1 Volts Li-Po battery. The specification and parameters of the quadrotor prototype are given in the Table 1.

\begin{tabular}{|c|c|c|c|}
\hline Parameter & Description & Value & Units \\
\hline$m$ & Mass & 0.835 & $\mathrm{Kg}$ \\
$d$ & Distance & 0.16 & $\mathrm{~m}$ \\
$J_{x}$ & Inertia in x-axis & $7.80 \times 10^{-3}$ & $\mathrm{Kg} \cdot \mathrm{m}^{2}$ \\
$J_{y}$ & Inertia in y-axis & $7.80 \times 10^{-3}$ & $\mathrm{Kg} \cdot \mathrm{m}^{2}$ \\
$J_{z}$ & Inertia in z-axis & $10.22 \times 10^{-3}$ & $\mathrm{Kg} \cdot \mathrm{m}^{2}$ \\
$b_{m}$ & Proportionality Constant & 5106.8 & $\mathrm{~N} / \mathrm{s}$ \\
$k_{m}$ & Proportionality Constant & 342.4 & $\mathrm{~N} \cdot \mathrm{m} / \mathrm{s}$ \\
\hline
\end{tabular}

Table 1 The specification and parameters of the Quadrotor
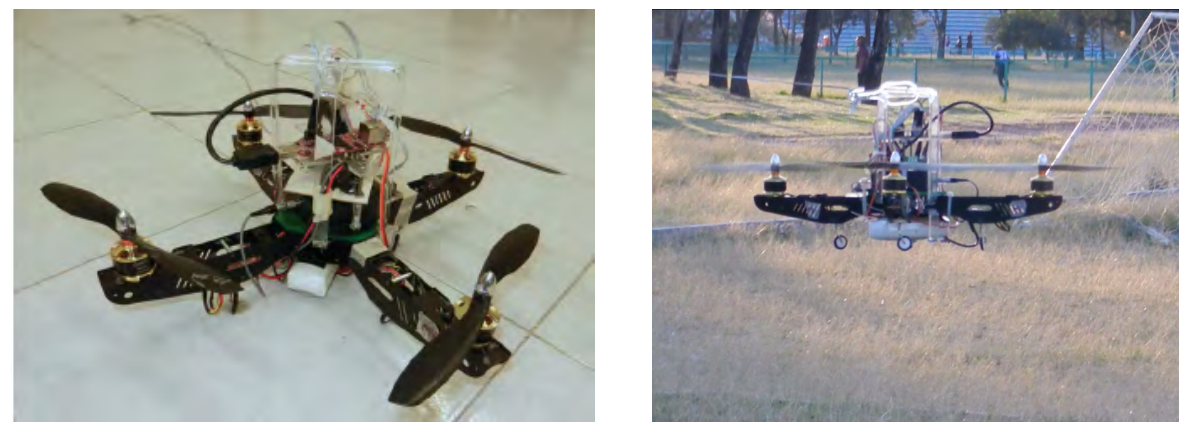

Fig. 3 The quadrotor mini-helicopter in flight

To evaluate the benefices of the control law defined in the corollary 1 , two experiments are performed. The objective is to bring the quadrotor from any initial orientation, sufficiently far from the desired attitude defined by $q_{d}=\left(\begin{array}{ll}1 & 0^{T}\end{array}\right)^{T}$ i.e. $\phi_{d}=\theta=0=\psi=0$ and hold it there by maintaining the angular velocity to zero. The desired thrust is taken as $T \geq m g=8.19 \mathrm{~N} \mathrm{such}$ that it guarantees a balance of the quadrotor's weight. Experiments were performed with the following gains: $K_{1}=\operatorname{diag}(1,1,1), K_{2}=\operatorname{diag}(2.5,2.5,2.5)$, $K_{3}=\operatorname{diag}(0.11,0.11,0.12)$. The value for the parameter $\sigma$ in the event function (8) determinates the frequency of events and it is fixed to 0.94 for these experiments.

In each cases, the first (top) plot shows the Euler angles (since they are more intuitive, however the control law uses quaternions) whereas angular velocities are provided in the second one. The third and fourth plots show the control torques and the Lyapunov function (one can see it decreases while the sys- 
tem is stabilized). Finally, the last (bottom) plots give the event function an event occurs when this function vanishes to zero, as defined in (8) - and a representation of the sampling instants $(1$ and 0 in the last plot mean the control is updated or it is kept constant respectively).

In the first experiment, the control capabilities are tested to stabilize the system, with initial conditions $\left(-21^{\circ}, 26.4^{\circ},-37^{\circ}\right)$. The results are depicted in Fig. 4 where the stabilization takes about 2.5 seconds. In the classical frame (time-triggered control), the control law should be updated 365 times for a span of 5 seconds, since the AHRS continuously provides the state at a frequency of $73 \mathrm{~Hz}$. With the proposed approach, one could note in Fig. 4(f) that some large intervals without any control update exist. Actually, the control law is updated only 72 times during the 5 seconds experimental time, which represents a reduction of $80.2 \%$ w.r.t. the classical frame. It is worth noting that this reduction in the number of updates, reduces the data exchange between AHRS, controller and actuators without sacrificing performance. Also, one could note in Fig. 4(e) that, whereas the event function only vanishes in theory (it could not become negative by construction), the implemented version becomes negative due to the AHRS sampling time. Indeed, an event can only be detected when some data are received and these data are only available every 0.0136 seconds.

In the second experiment, the robustness of the proposed controller towards disturbance rejection is tested. The disturbances along each axis (the three directions) are introduced in the system once achieved the attitude stabilization. The results are depicted in Fig. 5, where a disturbance is performed on roll, pitch and yaw at about 23, 33 and 46 seconds respectively. As one can see, the disturbances produce an error on both the angles and the angular velocities, see Fig. 5(a) and (b). As a consequence, the Lyapunov function in Fig. 5(d) is highly increased when a perturbation occurs. The event-triggered function vanishes or becomes negative in consequence and, as a result, the control law is updated more often to overcome the perturbations, see Fig. 5(c). In this experiment, the amount of samples needed for the event-triggered control for 55 seconds is 801 (instead of 4015 in the classical frame) which represents a reduction of $80 \%$.

\section{CONCLUSIONS}

The main contribution of this paper is the development and implementation of a nonlinear event-triggered feedback for the attitude stabilization of a quadrotor mini-helicopter. The attitude is parameterized using the unit quaternion. Firstly, it is proved the existence of a smooth Control Lyapunov Function for the attitude dynamics of the quadrotor. Then, an event-triggered static feedback is derived from the universal formula for event-triggered stabilization of general nonlinear systems affine in the control [24]. The control law ensures the asymptotic stability of the closed-loop system to the desired attitude. The approach is validated in real-time and the experiments show that the event 
driven controller reduces by $80 \%$ the communication load without deteriorating the closed-loop system performance. The proposed approach still has to be compare with other control schemes. However, to our best knowledge, this is the first time that a nonlinear event-triggered controller is applied for the attitude stabilization of an unmanned aircraft system.

\section{References}

1. E. A. Lee and S. A. Seshia, Introduction to Embedded Systems - A Cyber-Physical Systems Approach. Lee and Seshia, 2010.

2. P. Castillo, A. Dzul, and R. Lozano, "Real-time stabilization and tracking of a four-rotor mini rotorcraft," IEEE Trans. on Control Sytems Technology, vol. 12(4), pp. 510-516, 2004.

3. S. Bouabdallah, A. Noth, and R. Siegwart, "PID vs LQ control techniques applied to an indoor micro quadrotor," in Proc. of the IEEE/RSJ Conference on Intelligent Robots and Systems, vol. 3, 2004, pp. 2451-2456.

4. S. Bouabdallah and R. Siegwart, "Backstepping and sliding-mode techniques applied to an indoor micro quadrotor," 2005, pp. 2247-2252

5. N. Guénard, T. Hamel, and V. Moreau, "Dynamic modelling and intuitive control strategy for an X4-Flyer," in $5^{\text {th }}$ IEEE International Conference on Control and Automation, ICCA'05, vol. 1, Budapest, Hungary, 2005, pp. 141-146.

6. A. Tayebi and S. McGilvray, "Attitude stabilization of a VTOL quadrotor aircraft," IEEE Transactions on Control Systems Technology, vol. 14, no. 3, pp. 562-571, 2006.

7. P. Castillo, P. Albertos, P. Garcia-Gil, and R. Lozano, "Simple real-time attitude stabilization of a quad-rotor aircraft with bounded signals," in 45th IEEE Conference on Decision and Control, San Diego, CA, USA, 2006, pp. 1533-1538.

8. J. Guerrero-Castellanos, N. Marchand, A. Hably, S. Lesecq, and J. Delamare, "Bounded attitude control of rigid bodies: Real-time experimentation to a quadrotor minihelicopter," Control Engineering Practice, vol. 19, no. 8, pp. 790-797, 2011.

9. J. J. Corona-Sánchez and H. Rodríguez-Cortés, "Trajectory tracking control for a rotary wing vehicle powered by four rotors," Journal of Intelligent and Robotic Systems, vol. 70, no. 1-4, pp. 39-50, 2013.

10. K. Åström and B. Wittenmark, Computer Controlled Systems, 3rd Edition. Prentice Hall, 1997.

11. T. Chen and B. Francis, Optimal sampled-data control systems. Springer-Verlag, Berlin, Germany, 1995

12. A. Chemori and N. Marchand, "Global discrete time stabilization of the pvtol aircraft based on a fast predictive controller," in IFAC World Congress, 2008.

13. D. Nešić and A. Teel, "A framework for stabilization of nonlinear sampled-data systems based on their approximate discrete-time models," IEEE Trans. on Automatic Control, vol. 49 , no. 7 , pp. 1103-1122, 2004.

14. D. Nešić and L. Grüne, "Lyapunov-based continuous-time nonlinear controller redesign for sampled-data implementation," Automatica, vol. 41, no. 7, pp. 1143-1156, 2005.

15. K.-E. Årzén, "A simple event-based PID controller," in Preprints of the 14th World Congress of IFAC, Beijing, P.R. China, 1999

16. J. Sandee, W. Heemels, and P. van den Bosch, "Event-driven control as an opportunity in the multidisciplinary development of embedded controllers," in Proc. of the IEEE American Control Conference (ACC), 2005, pp. 1776-1781.

17. S. Durand and N. Marchand, "Further results on event-based PID controller," in Proc. of the European Control Conference (ECC), 2009.

18. J. Sánchez, M. Guarnes, S. Dormido, and A. Visioli, "Comparative study of eventbased control strategies: An experimental approach on a simple tank," in Proc. of the European Control Conference (ECC), 2009.

19. W. Heemels, J. Sandee, and P. van den Bosch, "Analysis of event-driven controllers for linear systems," International journal of control, vol. 81, pp. 571-590, 2009. 
20. J. Lunze and D. Lehmann, "A state-feedback approach to event-based control," Automatica, vol. 46, pp. 211-215, 2010.

21. K. Åström and B. Bernhardsson, "Comparison of Riemann and Lebesque sampling for first order stochastic systems," in Proc. of the IEEE Conference on Decision and Control (CDC), 2002.

22. M. Velasco, P. Martí, and E. Bini, "On Lyapunov sampling for event-driven controllers," in Proc. of the IEEE Conference on Decision and Control (CDC), 2009.

23. P. Tabuada, "Event-triggered real-time scheduling of stabilizing control tasks," IEEE Trans. on Automatic Control, vol. 52, no. 9, pp. 1680-1685, 2007.

24. N. Marchand, S. Durand, and J. F. Guerrero-Castellanos, "A general formula for eventbased stabilization of nonlinear systems," IEEE Trans. on Automatic Control, vol. 58, no. 5, pp. 1332-1337, 2013.

25. M. Lemmon, "Event-triggered feedback in control, estimation, and optimization," in Networked Control Systems Lecture Notes, ser. Control and Information Sciences Networked Control Systems, A. Bemporad, M. Heemels, and M. Johansson, Eds. SpringerVerlag, 2010, vol. 405, pp. 293-358.

26. J. Téllez-Guzmán, J. Guerrero-Castellanos, S. Durand, N. Marchand, and L. Maya, "Event-based LQR control for attitude stabilization of a quadrotor," in Proc. of the IFAC Latinoamerican Control Conference (CLCA), 2012, pp. 1-6.

27. A. Karimoddini, H. Lin, B. Chen, and T.-H. Lee, "Developments in hybrid modeling and control of unmanned aerial vehicles," in Proc.of the IEEE International Conference on Control and Automation (ICCA), 2009, pp. 228-233.

28. T. J. Koo, F. Hoffmann, H. Shim, B. Sinopoli, and S. Sastry, "Hybrid control of an autonomous helicopter," in Proc.of the IFAC Workshop on montion control, 1998, pp. $285-290$.

29. E. D. Sontag, "Clocks and insensitivity to small measurement errors," ESAIM Control, Optimisation and Calculus of Variations, vol. 4, pp. 537-557, 1999.

30. M. D. Shuster, "A survey of attitude representations," Journal of the astronautical sciences, vol. 41, no. 4, pp. 439-517, 1993

31. P. Pounds, R. Mahony, P. Hynes, and J. Roberts, "Design of a four-rotor aerial robot," in Australian Conference on Robotics and Automation, 2002.

32. S. Bhat and D. Bernstein, "A topological obstruction to global asymptotic stabilization of rotational motion and the unwinding phenomenon," Systems \& Control Letters, vol. 39, pp. $63-70,2000$.

33. N. A. Chaturvedi, M. K. Sanyal, and N. H. McClamroch, "Rigid-body attitude control," IEEE Control Systems Magazine, vol. 31, no. 3, pp. 30-51, June 2011. 


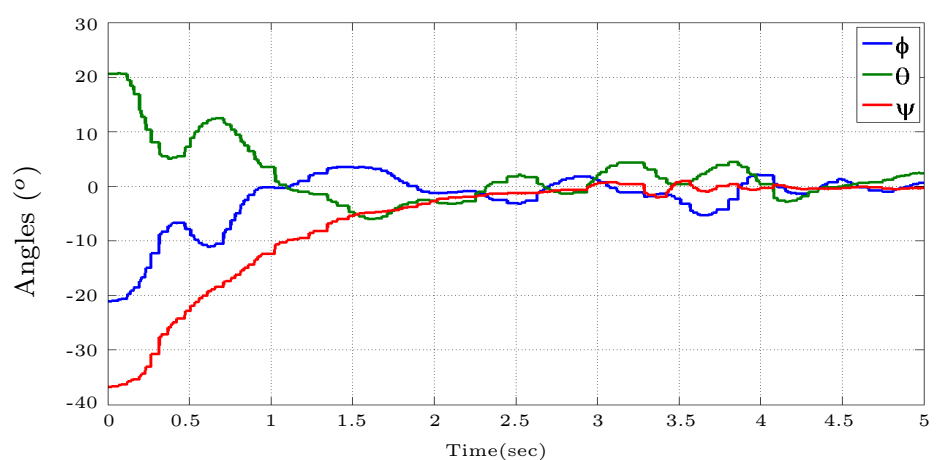

(a) Quadrotor attitude

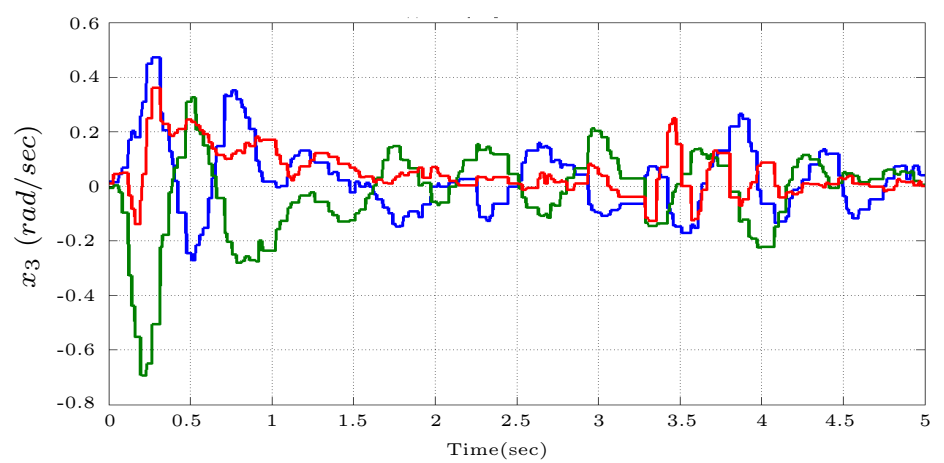

(b) Angular velocities

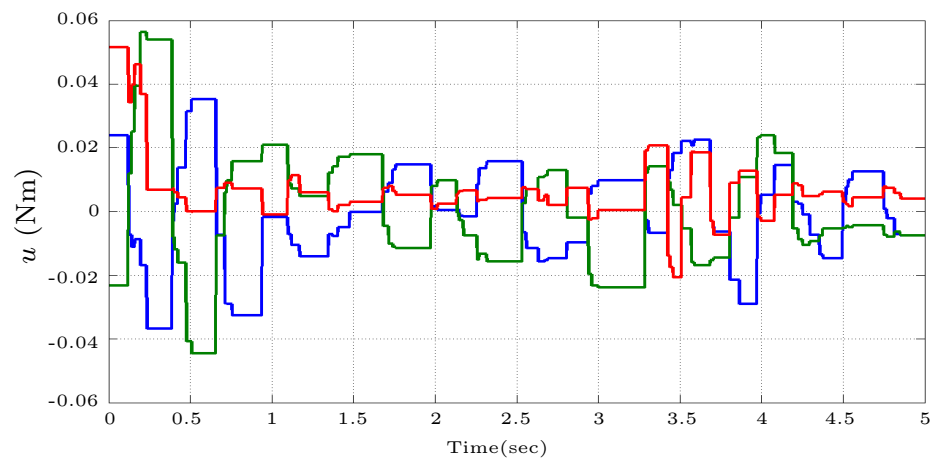

(c) Control

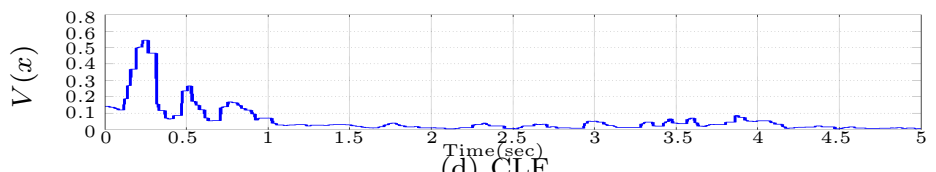

(d) $\mathrm{CLF}$

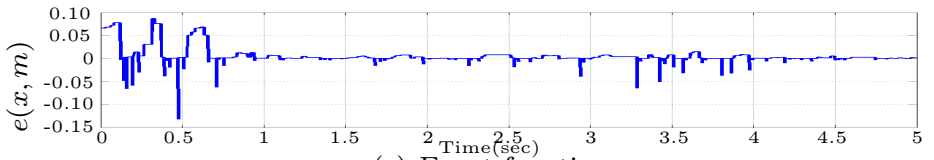

(e) Event function

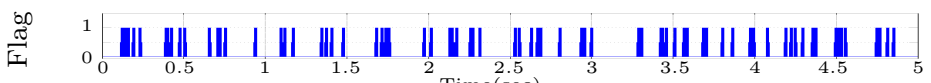

(f) Sampling instant times

Fig. 4 Stabilization to the origin of the quadrotor. 


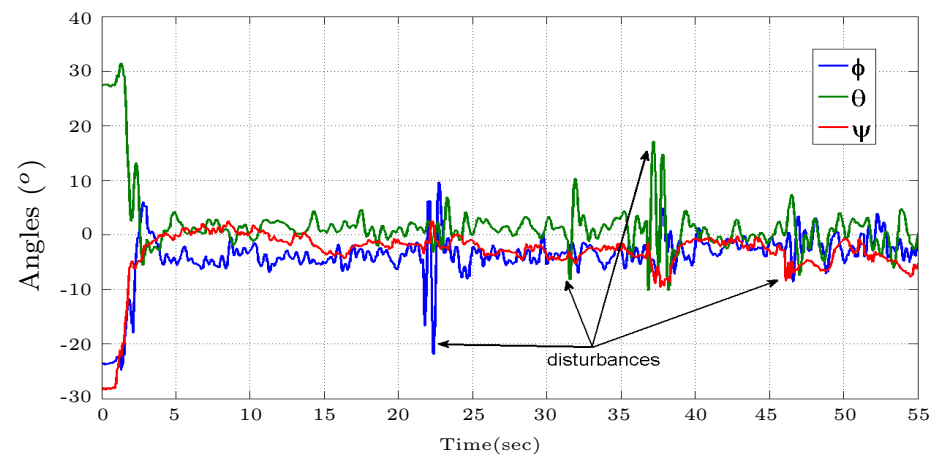

(a) Quadrotor attitude

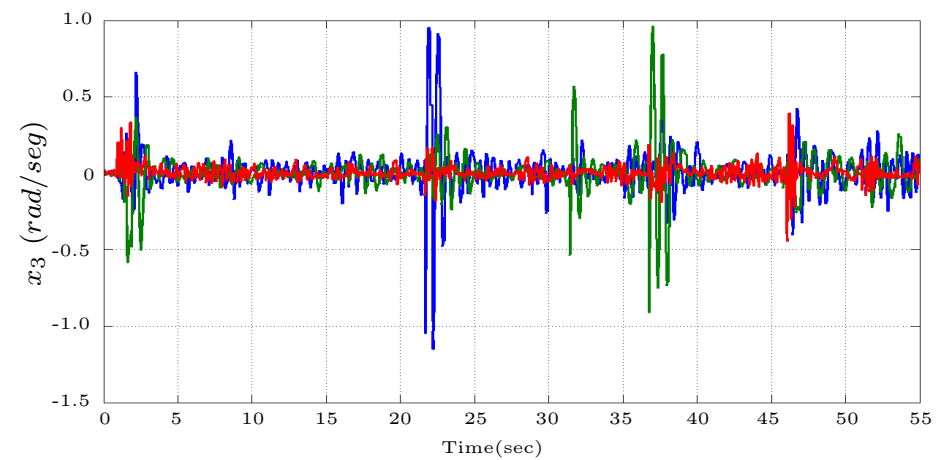

(b) Angular velocities

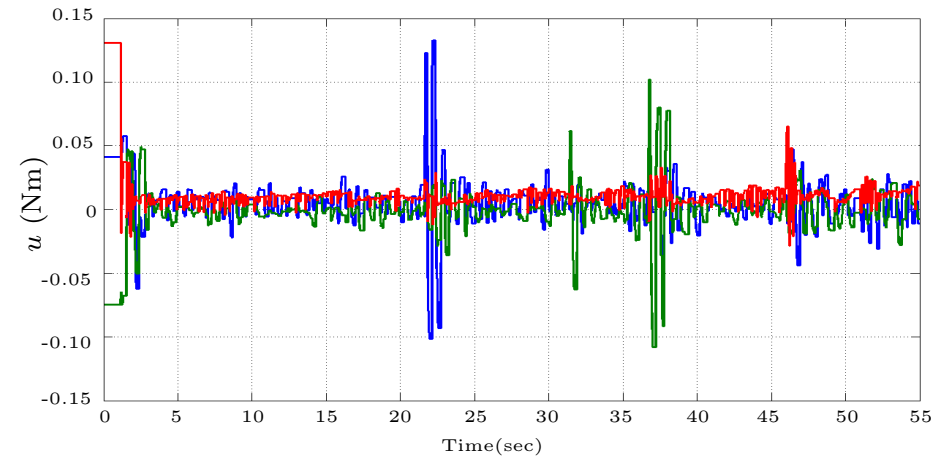

(c) Control

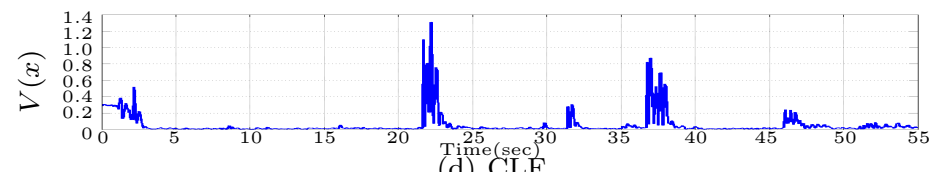

(d) CLF

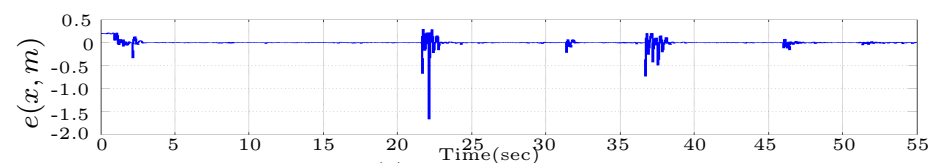

(e) Eviment function

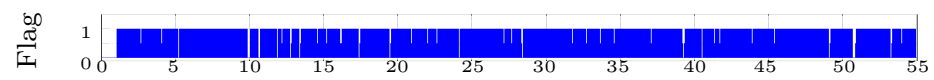

(f) Sampling instant times

Fig. 5 Robustness of the event-based control no-lineal to disturbances. 